Return to the Manage Active Submissions page at http://spie.org/app/submissions/tasks.aspx and approve or disapprove this submission. Your manuscript will not be published without this approval. Please contact author_help@spie.org with any questions or concerns.

\title{
NPP VIIRS On-Orbit Calibration and Characterization Using the Moon
}

\author{
J. Sun ${ }^{\mathrm{a}}$, X. Xiong ${ }^{\mathrm{b}}$, and J. Butler ${ }^{\mathrm{b}}$ \\ ${ }^{a}$ Sigma Space Corp., 4600 Forbes Boulevard, Lanham, Maryland 20706 \\ ${ }^{\mathrm{b}}$ Sciences and Exploration Directorate, NASA/GSFC, Greenbelt, MD 20771
}

\begin{abstract}
The Visible Infrared Imager Radiometer Suite (VIIRS) is one of five instruments on-board the Suomi National Polarorbiting Partnership (NPP) satellite that launched from Vandenberg Air Force Base, Calif., on Oct. 28, 2011. VIIRS has been scheduled to view the Moon approximately monthly with a spacecraft roll maneuver after its NADIR door open on November 21, 2011. To reduce the uncertainty of the radiometric calibration due to the view geometry, the lunar phase angles of the scheduled lunar observations were confined in the range from $-56^{\circ}$ to $-55^{\circ}$ in the first three scheduled lunar observations and then changed to the range from $-51.5^{\circ}$ to $-50.5^{\circ}$, where the negative sign for the phase angles indicates that the VIIRS views a waxing moon. Unlike the MODIS lunar observations, most scheduled VIIRS lunar views occur on the day side of the Earth. For the safety of the instrument, the roll angles of the scheduled VIIRS lunar observations are required to be within $\left[-14^{\circ}, 0^{\circ}\right]$ and the aforementioned change of the phase angle range was aimed to further minimize the roll angle required for each lunar observation while keeping the number of months in which the moon can be viewed by the VIIRS instrument each year unchanged. The lunar observations can be used to identify if there is crosstalk in VIIRS bands and to track on-orbit changes in VIIRS Reflective Solar Bands (RSB) detector gains. In this paper, we report our results using the lunar observations to examine the on-orbit crosstalk effects among NPP VIIRS bands, to track the VIIRS RSB gain changes in first few months on-orbit, and to compare the gain changes derived from lunar and SD/SDSM calibration.
\end{abstract}

Keywords: NPP, VIIRS, RSB, Crosstalk, Lunar Irradiance, Radiometric Calibration

\section{INTRODUCTION}

The Visible Infrared Imager Radiometer Suite (VIIRS) ${ }^{1}$ is one of five instruments on-board the Suomi National Polarorbiting Partenership (NPP) satellite that was successfully launched on October 28, 2011. VIIRS has 22 spectral bands covering a spectral range from 0.412 to $12.013 \mu \mathrm{m}$. Among them, fourteen are Reflective Solar Bands (RSB), seven are thermal emissive bands (TEB), and one is a day/night band (DNB). Like MODIS, the VIIRS RSB are calibrated on orbit with an on-board Solar Diffuser (SD) with its degradation tracked by a Solar Diffuser Stability Monitor (SDSM). ${ }^{2-3}$ VIIRS has also been scheduled to view the moon monthly through its Space View (SV) since launch. The Moon is a very stable radiometric reference in the visible and near-infrared spectral regions ${ }^{4-5}$ and can be used to track the VIIRS RSB on-orbit change. The moon has been widely used to calibrate the $\mathrm{RSB}^{6,7}$ and characterize both RSB and TEB for MODIS instruments ${ }^{8-11}$ as well as other NASA Earth Observing System's remote sensors. ${ }^{12-13}$ For VIIRS, the Angle of Incidence (AOI) of the SV is exactly the same as that of the SD. Thus, the lunar observation and SD calibration track the VIIRS RSB gain change at the same AOI. In principle, they should provide the same on-orbit gain variation for all RSB. The lunar observation can also be used to characterize both VIIR RSB and TEB crosstalk effects and other properties. ${ }^{8-11}$

There have already been six scheduled lunar observations since the NPP VIIRS launch. Since the lunar irradiance is strongly dependent on view geometry, the lunar observations have been carefully selected and scheduled. The main parameters used to select the lunar observations are lunar phase angle defined as the angle formed by the Sun, the moon, and the sensor, and the roll angle. ${ }^{6,7}$ The former is limited to a small range in order to reduce the potential error in the correction of the lunar view geometry effect on the measured lunar irradiance. The latter is kept as small as possible to avoid any instrument safety concerns. The phase angle range has changed from $\left[-56^{\circ},-55^{\circ}\right]$ to $\left[-50.5^{\circ}, 51.5^{\circ}\right]$ since the first scheduled lunar observation to reduce the frequency of facing the safety constraints. The lunar observation data collection has also been changed from the SV sector to Earth View (EV) sector due to the small size of the SV. The

8510 - 54 V. 2 (p.1 of 9) / Color: No / Format: Letter / Date: 8/2/2012 3:17:06 PM 
Return to the Manage Active Submissions page at http://spie.org/app/submissions/tasks.aspx and approve or disapprove this submission. Your manuscript will not be published without this approval. Please contact author_help@spie.org with any questions or concerns.

sector change is called sector rotation. With sector rotation, the VIIRS instrument still views the moon at its SV scan angle but lunar observation data are stored in EV sector. Since there is no a real SV port for VIIRS, the sector rotation actually increases the "effective" size of the SV. With scheduled lunar observations, the VIIRS crosstalk effect has been investigated, and it was found that there is no observable crosstalk effect in both VIIRS RSB and TEB. We have also calculated the lunar irradiance and lunar calibration coefficients (or F factors). The calibration coefficients are inversely proportional to the gains at the AOI of the SV or SD. They show that the on-orbit gain degradation is strongly wavelength dependent. The largest on-orbit gain degradation occurs for bands I2 $(865 \mathrm{~nm})$ and M7 $(865 \mathrm{~nm})$ while band M1 (412 nm) and other short wavelength bands have much smaller gain degradation since launch. This is very different from MODIS RSB early mission gain degradation, where the short wavelength bands have larger gain degradation. The unexpectedly large gain degradation in VIIRS NIR bands has been identified to be due to a $\mathrm{WO}_{3}$ contamination on the mirror surface of the Half Angle Mirror (HAM). The on-orbit gain changes derived form the lunar observations for the RSB are also compared to those calculated from the SD/SDSM calibration. They agree with each other very well.

\section{LUNAR OBSERVATIONS}

\subsection{Lunar Observation Scheduling}

VIIRS views the Moon through its SV port. With the instrument coordinate system defined as the $Z$ - and $X$-axis in the nadir and track directions, respectively, at nominal attitude, the SV is located in the YZ-plane with an offset angle of $24.325^{\circ}$ relative to the $Y$-axis (measured from the center of the SV port). ${ }^{16}$ The size of the SV sector is about $0.85^{\circ}$. Therefore the view through the SV port forms a cone with a vertex angle of $0.85^{\circ}$. As VIIRS circles in its orbit, the cone forms an angular vertex. The Moon is viewed whenever it passes into this angular vertex. A roll maneuver is a spacecraft rotation around the $\mathrm{X}$-axis, the track direction. With a roll maneuver, the angular vertex ring formed by the view cone of the SV can change its size. For VIIRS, the roll angle is always negative and the roll maneuver reduces the size of the angular vertex as shown in Figure 1. Thus, a roll maneuver does not increase the lunar observation opportunities of the VIIRS instrument. Without a roll maneuver, VIIRS can view the Moon for about nine months every year. With a roll maneuver, the number of months in which the Moon can be viewed by the VIIRS is still the same.

The lunar irradiance observed by a remote sensor strongly depends on viewing geometry: The Sun-Earth distance, the sensor-Moon distance, the phase angle formed by the Sun, Moon, and the sensor, and the lunar librations. ${ }^{7}$ The effects of the first two items can be described analytically. The effects of the last two items are wavelength-dependent and difficult to describe. The effect of the lunar phase angle is known to be much larger than that of the lunar librations. To minimize the errors due to geometric effects, lunar views used for lunar calibration are selected such that the lunar phase angle is kept within a limited range. The period of VIIRS orbit is about 100 minutes and the change in lunar phase angle in one-orbit is about $0.87^{\circ}$. Thus the phase angle difference between two consecutive possible views (orbits) is less than $1^{\circ}$, and the phase angle selection range can therefore be as small as $1^{\circ}$. We originally chose $\left[-55.5^{\circ},-54.5^{\circ}\right]$ for VIIRS as the acceptable phase angle range, where the phase angle is defined to be negative for a waxing Moon and positive for a waning Moon. Later, the range was changed to $\left[-51.5^{\circ},-50.5^{\circ}\right]$ to minimize the roll angles required for the scheduled lunar observations which will be discussed in more detail later.

The NPP VIIRS lunar observations with phase angles in the selected range can only occur in instrument daytime. ${ }^{15}$ This is different from MODIS scheduled lunar observations, which occur in instrument nighttime since the MODIS SV forms an annual vertex. ${ }^{6,13}$ Thus, there are more safety concerns for VIIRS roll maneuvers than for MODIS roll maneuvers. The roll angle for the VIIRS roll maneuvers is limited to $-14^{\circ}$ to $0^{\circ}$. This range is smaller than the roll angle range used for MODIS scheduled lunar observations, which is from $-20^{\circ}$ to $0^{\circ}$. With the roll angle limited to the range from $14^{\circ}$ to $0^{\circ}$ and the phase angle in $\left[-56^{\circ},-55^{\circ}\right]$, the VIIRS instrument can view the Moon in nine months each year. However, there are still cases among the lunar observations which violate the constraint imposed by the thermal keep-out zone $(\mathrm{KOZ})$ for the cryo-radiator, which must be protected from any exposure to sunlight. Violation can be avoided by limiting the roll angle to an even smaller range. To reduce the roll angle required and to keep the number of months in which VIIRS can view the Moon in a selected narrow phase angle range constant, the lunar phase angle range was changed to $\left[-51.5^{\circ},-50.5^{\circ}\right]$ in April 2012.

So far, there have been six scheduled and implemented lunar observations, which are listed in Table 1. The first two columns are days and times for the scheduled and implemented lunar observation. The third column lists the required

8510 - 54 V. 2 (p.2 of 9) / Color: No / Format: Letter / Date: 8/2/2012 3:17:06 PM 
Return to the Manage Active Submissions page at http://spie.org/app/submissions/tasks.aspx and approve or disapprove this submission. Your manuscript will not be published without this approval. Please contact author_help@spie.org with any questions or concerns.

and implemented roll angles except for last lunar observation on May 31, 2012 when no maneuver was required. The fourth column gives the lunar phase angles. For the lunar observations in January and February 2012, there was an attempt to keep the phase angles in the range from $-56^{\circ}$ to $-55^{\circ}$. However, as there were two lunar observations scheduled in February, only one of them could be kept in the phase angle range. Since April 2012, the phase angle range has been changed to $-51.5^{\circ}$ to $-50.5^{\circ}$ due to instrument safety concerns as aforementioned. The second May 2012 lunar observation was selected because a roll maneuver was unnecessary, even though its phase was not within the selected range. The roll angle in the table for this lunar observation was the roll angle required if a roll maneuver had been implemented. However, for this lunar observation, no roll maneuver was implemented. The last column lists the sector used to record the lunar view data for each observation. For the first three lunar observations, the lunar images can be found in the data of the SV sector while for last three lunar views they can only be found in EV sector.

The moon can intrude in the VIIRS SV for about nine months every year. In each such month, there are four intrusions. Two occur at instrument day side and two happen at instrument night side. These lunar intrusions are called unscheduled lunar observations. In most cases, VIIRS observes a partial moon in these unscheduled lunar observations. Furthermore, their phase angles are distributed over a very large range. In this report, we focus only on scheduled lunar observations.

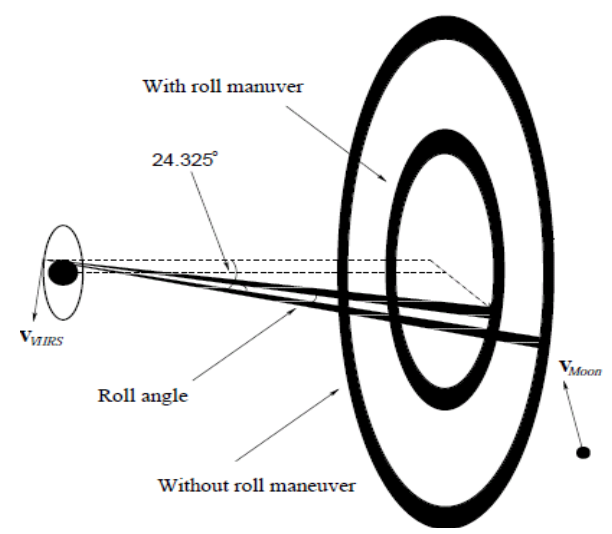

Table 1. Scheduled VIIRS lunar observations.

\begin{tabular}{|c|c|c|c|c|}
\hline M/D/ Y & H MS & Rd_ang & SMMV & Sector \\
\hline $1 / 4 / 2012$ & $8: 48: 53$ & -9.49 & -55.41 & SV \\
\hline $2 / 3 / 2012$ & $4: 21: 32$ & -5.452 & -56.19 & SV \\
\hline $2 / 3 / 2012$ & $6: 03: 34$ & -5.2794 & -55.38 & SV \\
\hline $4 / 2 / 2012$ & $23: 05: 11$ & -3.9893 & -51.24 & EV \\
\hline $5 / 2 / 2012$ & $10: 20.06$ & -3.225 & -50.92 & EV \\
\hline $5 / 31 / 2012$ & $14: 47: 14$ & -0.0806 & -5297 & EV \\
\hline
\end{tabular}

Figure 1. A schematic diagram for a VIIRS lunar observation.

\subsection{Lunar Data and Images}

The SV size is $0.85^{\circ}$ as mentioned above, which corresponds to 48 samples for M bands and 96 samples for I bands. The Moon size is about $0.5^{\circ}$, which corresponds to about 26 samples for $\mathrm{M}$ bands and 52 samples for I bands. Figure 2 shows the distribution of the VIIRS bands on the Focal Plane Assemblies (FPA). Different bands are located at different locations on the FPA, and they view different spots on any target at any given time. The corresponding differences on view target due to the location differences on the FPA are shown in the plot, which vary from - 20 to $21 \mathrm{M}$ band samples. Then for every band on the FPA to observe the whole moon, the size of the SV should be at least $67 \mathrm{M}$ band samples, which is larger than the actual size of VIIRS SV. If co-registration were applied to VIIRS SV sector, the real size of the SV would be at least $67 \mathrm{M}$ band samples and the $48 \mathrm{M}$ band samples and $96 \mathrm{I}$ band samples in the SV sector would be realigned data. Then the SV sector could provide a full lunar image for each band on the FPA. Unfortunately, this is not the case for the VIIRS SV sector. Co-registration was not applied to the SV sector, which was discovered during the first VIIRS lunar observation, an unscheduled lunar observation in December 2012, and was confirmed in first scheduled lunar observation on January 4, 2012.

Figure 3 shows a lunar image observed by band I1 on January 4, 2012. The size of the moon is smaller than the full 60 samples. This is because the moon is not a full moon and part of the lunar surface observed by the band is dark. Figure 4 shows the lunar image observed by the band M6. As expected, resolution of band M6 is different from that of band I1. Compared to the lunar image of band I1 in Figure 3, the lunar image of band M6 has moved to right side of the SV. This indicates that co-registration is not applied to the SV sector. Then it is impossible for all bands to see the whole moon through the SV as discussed above. Figure 5 shows the lunar image observed by band M1, which demonstrates that the Moon was only partially observed by band M1 during the lunar observation on January 4, 2012. This further confirmed 
Return to the Manage Active Submissions page at http://spie.org/app/submissions/tasks.aspx and approve or disapprove this submission. Your manuscript will not be published without this approval. Please contact author_help@spie.org with any questions or concerns.

that co-registration is not applied to the SV sector and the bands on the edge of the FPA may see a partial moon during a lunar calibration through the SV. Then such a lunar calibration cannot be used to track the gain change for all RSB.

The two lunar observations in two continuous orbits with tuned roll angles were applied in the February 2012 lunar calibration to overcome the difficulties that occurred in the first scheduled lunar observation. The lunar observation in the first orbit (2/3/20120 4:21:23) focused on the bands on the left side of the FPA and ensured they viewed the whole moon while the second lunar observation (2/3/2012 6:03:34) emphasized the bands on right side of the FPA and ensured they viewed the whole moon. This was the first time that all bands viewed the whole moon even though the images were not located at the center of the SV. Two lunar observations for one lunar calibration are not a good choice. Furthermore the small size of the SV limits the capability of using the lunar observation for other investigations such as crosstalk analysis, which was a serious issue in VIIRS prelaunch characterization and calibration. Another approach with a sector rotation was proposed and implemented in the following scheduled lunar observations. With the sector rotation, the EV sector data collection is moved earlier in scan such that the observed data during the nominal SV scan angles are collected in the EV sector. Since the VIIRS SV has no a real port such as the SV port MODIS has, the sector rotation greatly increase the size of the SV and enables the instrument to collect much more useful data. It also has coregistration applied such that all the lunar images observed by different bands are aligned at the center of the EV.

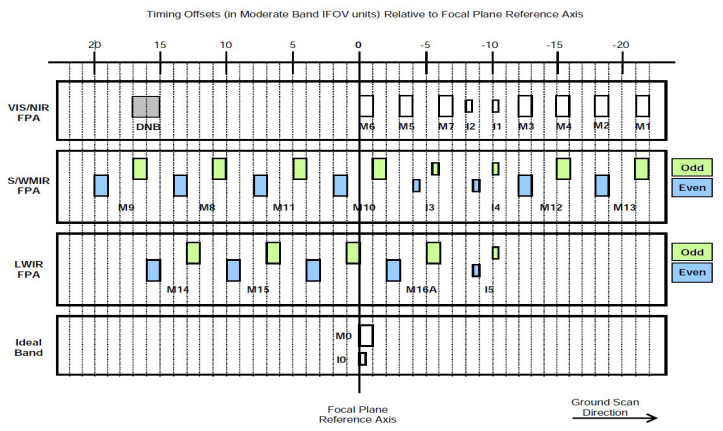

Figure 2. VIIRS Focal Plane Assemblies.

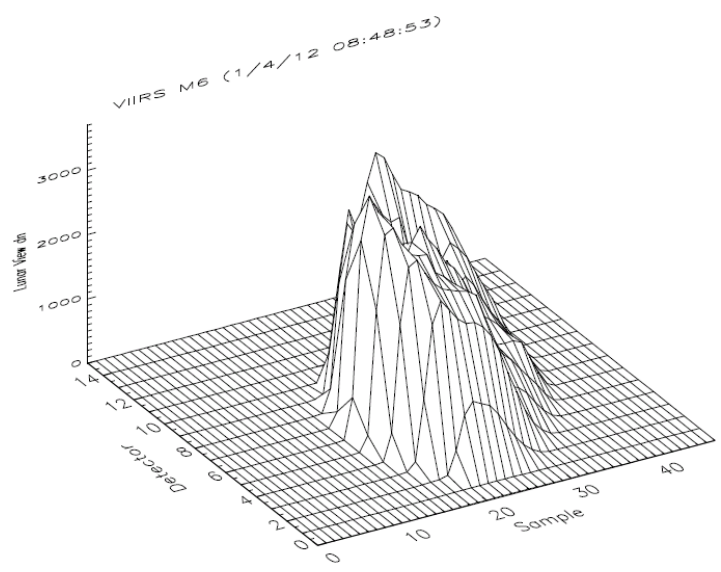

Figure 4. Lunar image observed by band M6 on January 4, 2012.

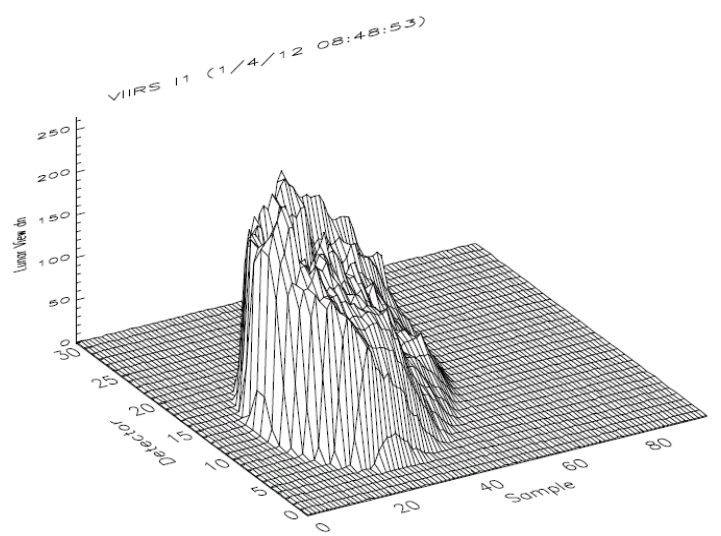

Figure 3. Lunar image observed by band I1 on January 4, 2012.

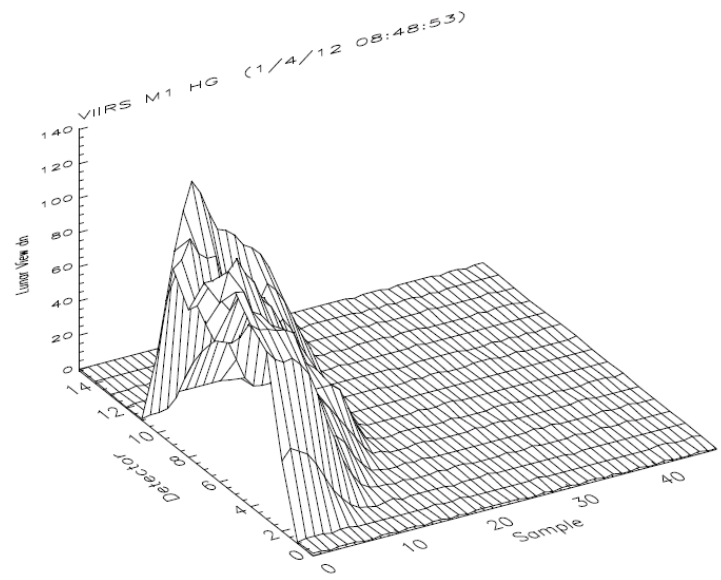

Figure 5. Lunar image observed by band M1 on January 4, 2012

An aggregation scheme is applied to VIIRS EV data. At the center of the EV, three detector footprints are aggregated to form a single VIIRS pixel. The scheme is applied to all $\mathrm{I}$ bands and single gain $\mathrm{M}$ bands. For dual gain $\mathrm{M}$ bands, the scheme is applied to calibration data but not the pre-calibrated instrument response. For lunar calibration, we only need the instrument response during a lunar observation. Thus the sector rotation does not change the resolution of the dual 
Return to the Manage Active Submissions page at http://spie.org/app/submissions/tasks.aspx and approve or disapprove this submission. Your manuscript will not be published without this approval. Please contact author_help@spie.org with any questions or concerns.

gain $\mathrm{M}$ bands, but it does reduce the resolution of the other bands. Figures 6 and 7 display the lunar images observed by bands I1 and M6 in April, 2012, respectively. I1 is an I band and M6 is a single-gain M band. Compared to the images in Figures 3 and 4, it is easy to see that the resolutions of the images in Figures 6 and 7 along the scan direction have been reduced by a factor of 3 .

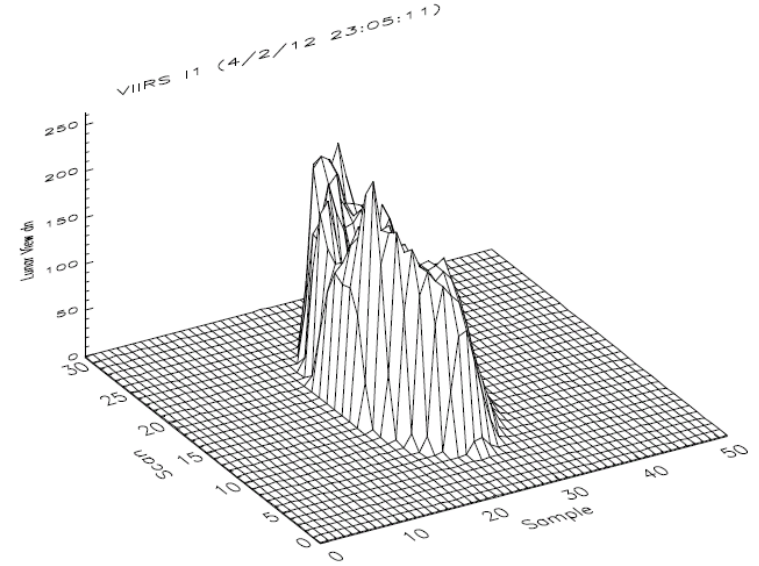

Figure 6. Lunar image observed by band I1 on April 2, 2012.

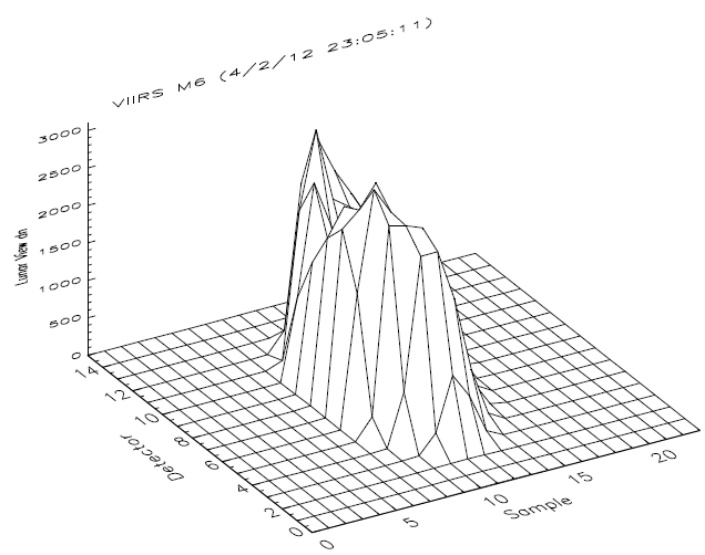

Figure 7. Lunar image observed by band M6 on April, 2012

It is well known that a lot of MODIS TEB as well as some of MODIS RSB are saturated when they view the moon. The saturation brings difficulties for MODIS lunar calibration and characterization. For NPP VIIRS, all reflective solar bands including VIS, NIR, and SWIR bands are not saturated. Therefore, the lunar calibration can be used to track their on-orbit gain change. For NPP VIIRS TEB, bands I4-I5 and M12, M13 (high gain), and M14-M16 are saturated during a lunar observation.

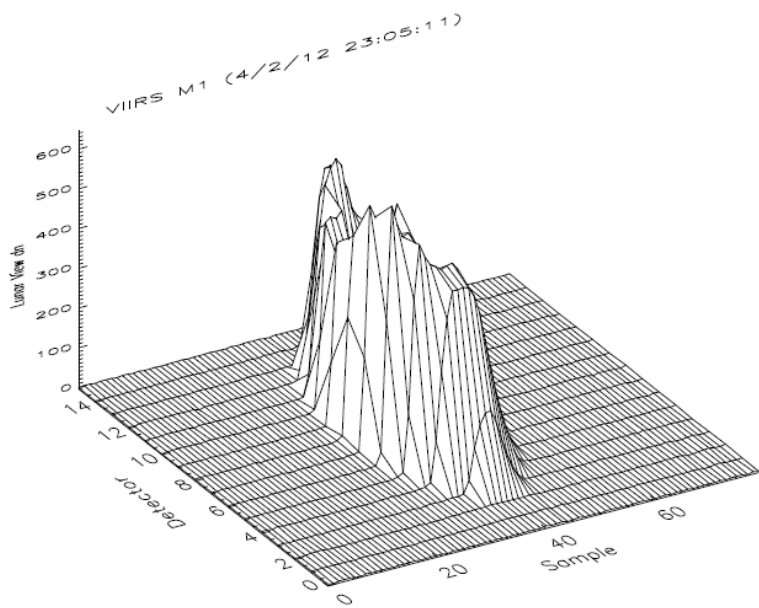

Figure 8. Lunar image observed by band M1 on April 2, 2012.

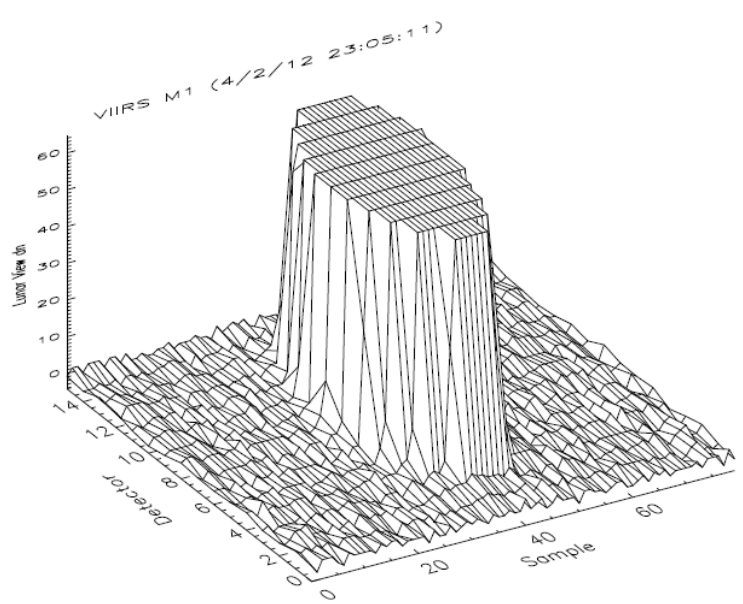

Figure 9. Lunar image observed by band M1 on April, 2012 with response larger than 60 excluded.

\section{VIIRS LUNAR CHARACTERIZATION AND CALIBRATION}

\subsection{Crosstalk Analysis}

Electronic crosstalk was a serious issue during prelaunch characterization and calibration for the NPP VIIRS instrument. Great effort was spent to measure and analyze the crosstalk effect among the bands on the same focal plane assembly. The crosstalk effect was found in almost all bands. The crosstalk analysis was a challenging task since the crosstalk 
Return to the Manage Active Submissions page at http://spie.org/app/submissions/tasks.aspx and approve or disapprove this submission. Your manuscript will not be published without this approval. Please contact author_help@spie.org with any questions or concerns.

coefficients derived from different measurements were different. The differences were thought to be caused by the light contamination. The Moon is a unique light source for crosstalk analysis. It has been applied to analyze MODIS crosstalk effect and derive crosstalk coefficients. ${ }^{9,10}$ The basic idea to use the Moon to analyze the crosstalk effect is that the crosstalk effect induces "ghost" images, the centers of which are shifted from the center of real lunar image. The amount of shift for a "ghost" image depends on the distance between the receiving band and sending on the FPA.

Figure 8 shows the lunar image observed by VIIRS band M1 on April 2, 2012. No "ghost" image can be seen in the plot. To see more clearly, the observed lunar image is plotted with a smaller scale in Figure 9, where responses larger than 60 are excluded. Still, no "ghost" image can be seen in the plot. As with band M1, no "ghost" image can be seen in their lunar images for all other bands except bands M14 $(8.55 \mu \mathrm{m})$ and M15 $(10.76 \mu \mathrm{m})$, which are thermal emissive bands. This indicates that the crosstalk effect seen in prelaunch measurements is not present in NPP VIIRS after it launched for most of the VIIRS bands. For bands M14 and M15, Figures 10 and 11 show their lunar images observed on April 2, 2012. As mentioned earlier, the two bands are saturated when they view the moon. In Figures 10 and 11, the response larger than 380 is excluded in order to clearly see if there is any "ghost" image. The two images demonstrate that there is unexpected response around the edge of the "real" lunar images. However, the unexpected response does not look like typical "ghost" images induced by electronic crosstalk. ${ }^{9,10}$ It could be near field response effect but further investigation is needed to identify the exact cause for the unexpected response seen in Figures 10 and 11.

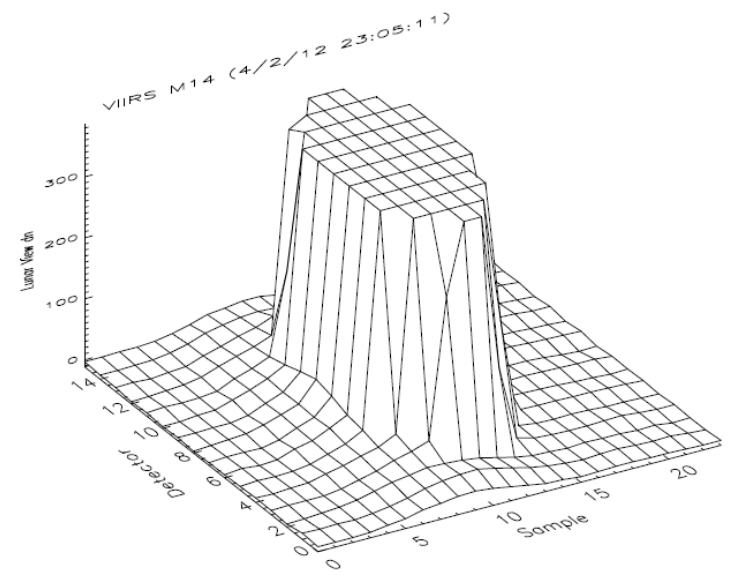

Figure 10. Lunar image observed by band M14 on April 2, 2012 with response larger than 380 excluded.

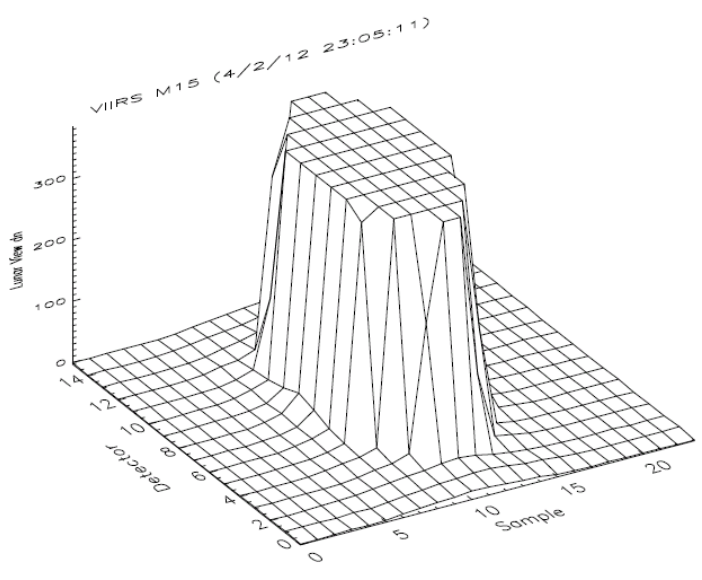

Figure 11. Lunar image observed by band M15 on April, 2012 with response larger than 380 excluded.

\subsection{Lunar Irradiance}

For MODIS RSB, the radiance is linearly related to the instrument response while for VIIRS RSB, a quadratic from is applied to convert the instrument's response to incident radiance. Then VIIRS RSB lunar radiance can be expressed as

$$
L(B, D, S, N)=F(B, D, M) L_{p l}(B, D, S, N) / R V S\left(B, M, \alpha_{S V}\right),
$$

where $B, D, S$, and $N$ are band number, detector number, sample index, and scan number, respectively, $M$ is the mirror side index of the HAM for scan $N, F(B, D, M)$ is the calibration coefficient which is also called the $\mathrm{F}$ factor, $\alpha_{S V}$ is the AOI of the SV with a value of $60.2^{\circ}, \mathrm{RVS}\left(\mathrm{B}, \mathrm{M}, \alpha_{S V}\right)$ is the Response Versus Scan angle (RVS), and

$$
L_{p l}(B, D, S, N)=c_{0}(B, D, M)+c_{1}(B, D, M) d n_{\text {Moon }}(B, D, S, N)+c_{2}(B, D, M) d n_{\text {Moon }}^{2}(B, D, S, N)
$$

is the radiance calculated with prelaunch calibration coefficients, $c_{0}(B, D, M), c_{1}(B, D, M), c_{2}(B, D, M)$ and the background subtracted instrument response, $d n_{\text {Moon }}(B, D, S, N)$.

The VIIRS RSB F factor is derived from on-orbit SD/SDSM calibration. The inverse of the coefficient tracks the degradation of the VIIRS optical system at AOI of the SD which is $60.2^{\circ}$ on the Half Angle Mirror (HAM). For a scan which fully covers the Moon, the lunar irradiance can be calculated by

8510 - 54 V. 2 (p.6 of 9) / Color: No / Format: Letter / Date: 8/2/2012 3:17:06 PM 
Return to the Manage Active Submissions page at http://spie.org/app/submissions/tasks.aspx and approve or disapprove this submission. Your manuscript will not be published without this approval. Please contact author_help@spie.org with any questions or concerns.

$$
I(B)=\sum_{D, S} L(B, D, S, N) w_{B},
$$

where $w_{B}=(0.742 \mathrm{~km}) *(0.259 \mathrm{~km})^{*} \mathrm{~g}_{\mathrm{B}} /(824 \mathrm{~km})^{2}$ is the solid angle (in steradians) of each pixel of the band B and $\mathrm{g}$ varies with band and the data mode. For M and I bands in SV sector, $g_{B}$ equals 1 and $1 / 4$, respectively. When the data is collected from the EV sector, the single gain $\mathrm{M}$ and $\mathrm{I}$ bands are in aggregated mode and then $g_{B}$ equals 1 for dual gain $\mathrm{M}$ bands, 3 for single gain $\mathrm{M}$ bands, and $3 / 4$ for I bands, respectively. The lunar irradiance can also be calculated from several scans which all fully cover the Moon, that is,

$$
I(B)=\sum_{D, S, N} L(B, D, S, N) w_{B} / N_{t},
$$

where $N_{t}$ is the number of the scans used in the calculation. The lunar irradiance can also be calculated from all scans which view the moon even though some of them may only partially observe the Moon as long as the oversampling effect is corrected.

Figure 12 shows the lunar irradiance for NPP VIIRS VIS bands. It is clearly seen that lunar irradiance is wavelength dependent and varies with view geometry. The variation of the lunar irradiance with the view geometry in the five months is about $20 \%$. This is mainly due to the Sun-Earth distance and sensor-Moon distance changes. The former can induce the lunar irradiance change as large as $27 \%$ while the latter can cause the lunar irradiance variation as large as $8 \%$. The next main factor for lunar irradiance variation is lunar phase angle. With the phase angle confined in a range with $1^{\circ}$ width, the phase variation can only induce a lunar irradiance change of as much as $2 \%$. However, the phase angle range change from $\left[-56^{\circ},-55^{\circ}\right]$ to $\left[-50.5^{\circ}, 51.5^{\circ}\right]$ may induce a lunar irradiance variation of $8 \%$. The lunar irradiance also depends on lunar librations but the impact of the lunar librations on the lunar irradiance is about $2 \%$. The VIIRS lunar irradiance has been compared to ROLO model predicted values. The differences between two sets of irradiance are around 3\% for band M1. For other bands, the differences vary from 5\% to $10 \%$.

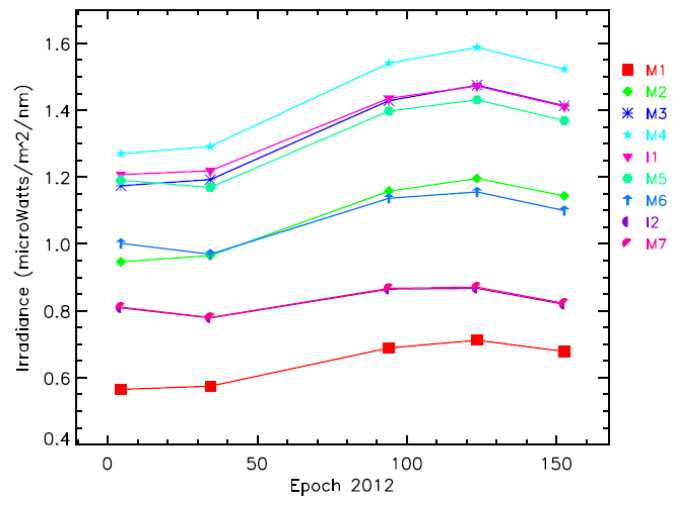

Figure 12. NPP VIIRS VIS bands lunar irradiance.

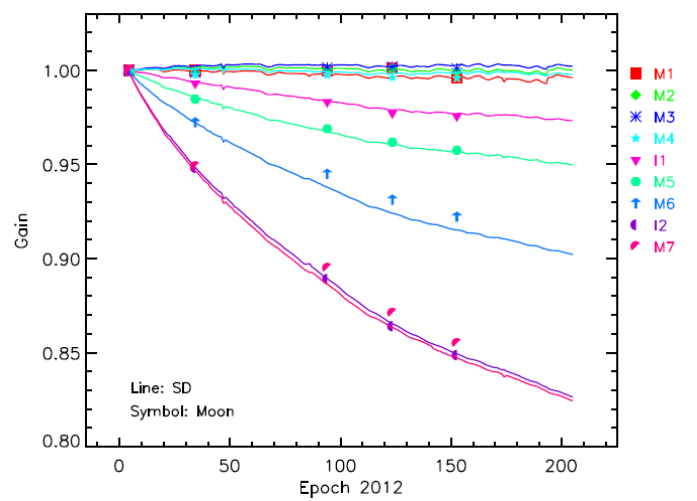

Figure 13. NPP VIIRS RSB F factors and lunar F factors.

\subsection{Lunar Calibration Coefficients}

VIIRS views the Moon from its SV as mentioned earlier, which has the same AOI as the SD although their optical paths on the HAM are the reverse of each other. Being a well studied target with a very stable reflectance in visible and nearinfrared spectral regions, the Moon can be used to track the VIIRS RSB gain change at the SD AOI. With the approximation that the detector difference is negligible, the detector averaged $\mathrm{F}$ factor can be expressed as

$$
F(B, M)=\frac{I(B) N_{t, M}}{\sum_{D, S, N} L_{p l}(B, D, S, N) \delta\left(M, M_{N}\right) / R V S\left(B, M, \alpha_{S V}\right)},
$$

where $N_{t, M}$ is the number of scans in the summation with HAM side M. To calculate the F factor, one has to know the lunar irradiance. The lunar irradiance depends on the view geometry, that is, Sun-Earth distance, sensor-moon distance, 
Return to the Manage Active Submissions page at http://spie.org/app/submissions/tasks.aspx and approve or disapprove this submission. Your manuscript will not be published without this approval. Please contact author_help@spie.org with any questions or concerns.

lunar phase angle, and lunar librations as mentioned earlier. The ROLO model can provide the predicted lunar irradiance. The absolute uncertainty of the prediction can be as large as $5 \%$ for short-wavelength bands and the uncertainty is even larger for NIR bands. However, the relative uncertainty of the irradiance predicted by the ROLO model over the entire view geometry is about $1 \%$. Since the lunar phase angle is confined to a selected region, the relative uncertainty of the lunar irradiance predicted by the ROLO model for VIIRS scheduled lunar observations should be smaller than $1 \%$. Thus, the lunar calibration may not provide accurate absolute $\mathrm{F}$ factors for VIIRS RSB but can provide relative $\mathrm{F}$ factors or the on-orbit gain change since the first lunar observation as demonstrated and confirmed by MODIS lunar calibration. ${ }^{6}$

For the relative F factor, Eq. (5) can be simplified and rewritten as

$$
f(B, M)=\frac{g(B) N_{t, M}}{\sum_{D, S, N} L_{p l}(B, D, S, N) \delta\left(M, M_{N}\right)},
$$

where $g(B)$ does not have to be an accurate lunar irradiance as long as it corrects the relative geometric effects. Since the AOI of the SD and the SV is the same, the lunar and the SD/SDSM calibration track the on-orbit gain change at the same AOI and, then, should provide the same calibration coefficients or gain changes when they are normalized at any given time. As with the lunar irradiance, the relative factor can also be calculated from all scans with the oversampling corrected. In this work, we focus on the calculation of the VIIRS RSB relative F factor using the lunar observations with scan which fully cover the lunar surface. Since the oversampling effect is event dependent, the number of scans which fully cover the lunar surface is different for different lunar observations. To simplify the calculation, we use the four scans around the center of the lunar observation, during which the instrument scans the center of the lunar surface, to calculate the relative $\mathrm{F}$ factor for each lunar calibration, two for each mirror side. To distinguish the $\mathrm{F}$ factor derived from the SD/SDSM calibration, the relative $\mathrm{F}$ factors obtained from the lunar calibration will be called lunar $\mathrm{F}$ factors in this work.

The gains, proportionally inverse to the F factors, derived for NPP VIIRS VIS bands from the scheduled lunar observations listed in Table 1 are shown in Figure 13 with symbols. For comparison, the corresponding gains derived from the SD/SDSM calibration are also drawn in the plots with lines. The gains are normalized at the time of the first scheduled lunar observation, respectively, for each band. From the plot, it can be seen that the gains form the two calibrations agree with each other very well. The largest difference occurs in bands M6 and M7, which is about 1\%. For other bands, the differences are less than $0.5 \%$. Among these bands, bands M7 $(865 \mathrm{~nm})$ and I2 $(865 \mathrm{~nm})$ have degraded most while band M1 (412 nm) has no observable degradation in last seven months. From the plot, it can be seen that the degradation is wavelength dependent and the degradation rate increases with wavelength. This is very different from MODIS RSB. For MODIS RSB, the degradation is also wavelength dependent but the largest degradation occurs at the band with shortest wavelength early in the mission. The unexpected VIIRS VIS band gain degradation is known to be caused by the $\mathrm{WO}_{3}$ contamination on the mirror surface of the HAM.

\section{SUMMARY}

The NPP VIIRS has been scheduled to view the Moon with a spacecraft roll maneuver on an approximately monthly basis since the beginning of 2012. To reduce the uncertainty of the radiometric calibration due to the view geometry, the lunar phase angles of the scheduled lunar observations were confined in the range from $-56^{\circ}$ to $-55^{\circ}$ in first three lunar observations and then changed to the range from $-51.5^{\circ}$ to $-50.5^{\circ}$ due to an instrument safety concerns. NPP VIIRS views the Moon through its SV and the lunar observation data were collected in the SV sector in first three scheduled lunar observations and then the data collection sector was changed to the EV sector. The VIIRS SV size is too small such that half of VIIRS bands only partially view the Moon during a lunar observation. The sector rotation enlarges the actual size of the SV and enables all bands view the whole Moon. Using the lunar observations, the crosstalk effect between different bands was analyzed and no evident crosstalk effect was found in all bands except bands M14 and M15. The unexpected response at the edge of the lunar images is not necessarily due to crosstalk. The lunar irradiance for these observations has been calculated. As expected, it is wavelength- and view geometry-dependent. The lunar calibration coefficients or F factors are also calculated from the lunar observations for VIIRS RSB. The on-orbit gain changes obtained from the coefficients are strongly wavelength dependent. The largest gain degradation occurs in NIR 
Return to the Manage Active Submissions page at http://spie.org/app/submissions/tasks.aspx and approve or disapprove this submission. Your manuscript will not be published without this approval. Please contact author_help@spie.org with any questions or concerns.

bands while the short wavelength bands have much smaller gain degradation. The gain changes derived from lunar observations for VIIRS RSB are compared to those obtained from the SD/SDSM calibration and they agree very well.

\section{ACKNOWLEDGEMENTS}

We would like to thank Tom Stone for providing the ROLO model predicted lunar irradiances which has been used as a view geometry correction factor in our lunar radiometric calibration. We appreciate Jon Fulbright and Zhipeng Wang for their helpful support and suggestions. Our thanks also go to Jeff Mclntire for his valuable comments.

\section{REFERENCES}

1. C. Schueler, J. E. Clement, P. Ardanuy, C. Welsh, F. DeLuccia, and H. Swenson, "NPOESS VIIRS sensor design overview", Proc. SPIE 4483, 11-23 (2001).

2. X. Xiong, J. Sun, W. Barnes, V. Salomonson, J. Esposito, H. Erives, and B. Guenther, "Multiyear on-orbit calibration and performance of Terra MODIS reflective solar bands", IEEE Trans. Geosci. Remote Sensing 45, 879889 (2007).

3. X. Xiong, J. Sun, X. Xie, W. Barnes, V. Salomonson, "On-Orbit Calibration and Performance of Aqua MODIS Reflective Solar Bands", IEEE Trans. Geosci. Remote Sensing 48, 535-546 (2010).

4. H. H. Kieffer, "Photometric stability of the lunar surface", Icarus 130, 323-327 (1997).

5. H. H. Kieffer and T. C. Stone, "The spectral irradiance of the Moon", Astronom. J. 129, 2887-2901 (2005).

6. J. Sun, X. Xiong, B. Guenther and W. L. Barnes, "Radiometric Stability Monitoring of the MODIS Reflective Solar Bands Using the Moon", Metrologia 40, S85-88, 2003.

7. J. Sun, X. Xiong, W. L. Barnes, and B. Guenther, "MODIS Reflective Solar Bands On-Orbit Lunar Calibration", IEEE Trans. Geosci. Remote Sens. 43, 2383-2393 (2007).

8. X. Xiong, J. Sun; K. Chiang, S. Xiong; and W. Barnes, "MODIS on-orbit characterization using the Moon", Proc. SPIE, 4881, 299-307, (2002).

9. J. Sun, X. Xiong, N. Che, and A. Angal, "Terra MODIS band 2 electronic crosstalk: cause, impact, and mitigation," Proc. SPIE, 7826, 78261Y, (2010). [doi: 10.1117/12.864738]

10. J. Sun, S. Madhavan, B. Wenny, and X. Xiong, "Terra MODIS band 27 electronic crosstalk: cause, impact, and mitigation", Proc. SPIE, 8176, 81760Z, (2011).

11. X. Xiong, J. Sun, S. Xiong, and W. L. Barnes, "Using the Moon for MODIS Spatial Characterization", Proc. SPIE, 5234, 480, (2003).

12. W. L. Barnes, X. Xiong, R. Eplee, J. Sun, and C. Lyu, "Use of the Moon for calibration and characterization of MODIS, SeaWiFS and VIRS, Earth Science Satellite Remote Sensing", J. Qu, W. Gao, M. Kafatos, R. Murphy, and V. Salomonson eds., Vol. 2, 98-119, Springer-Verlag (2006).

13. R. A. Barnes, R.E. Eplee, Jr., F.S. Patt, and C.R. McClain, "Changes in the radiometric stability of SeaWiFS determined from lunar and solar measurements", Appl. Opt., 38, 4649-4664 (1999).

14. J. Sun and X. Xiong, "Solar and lunar observation planning for Earth-observing sensor", Proc. SPIE, 8176, 817610 , (2011).

15. F. S. Patt, Eplee, R. E., Barnes, R. A., Meister, G., and Butler, J. J., "Use of the moon as a calibration reference for NPP VIIRS", Proc. SPIE 5882, 588215 (2005); doi:10.1117/12.618370

16. X. Xiong, J. Sun, and W. Barnes, "Intercomparison of On-Orbit Calibration Consistency Between Terra and Aqua MODIS Reflective Solar Bands Using the Moon", IEEE Geosci. Remote Sens. Lett. 5, 778-782, (2008). 